\title{
LA FORMA CANTABA EN LAS GRAMÁTICAS DE ESPAÑOL COMO LENGUA EXTRANJERA: PROPUESTAS TEÓRICAS Y CONTRASTE DE CORPUS
}

\author{
THE FORM CANTABA IN THE GRAMMARS OF SPANISH AS \\ A FOREIGN LANGUAGE: THEORETICAL PROPOSALS AND \\ CONTRAST OF CORPUS
}

\author{
Alfonso ZAMORANO AGUILAR \\ Universidad de Córdoba \\ azamorano@uco.es \\ María MARTÍNEZ-ATIENZA \\ Universidad de Córdoba \\ mmartinezatienza@uco.es
}

Resumen: El objetivo de este artículo es analizar el tratamiento que recibe el pretérito imperfecto (forma cantaba) en un corpus constituido por 24 gramáticas de español como lengua extranjera (ELE) de distintos niveles y lenguas publicadas entre 1988 y 2015. Estudiamos, en particular, el marco teórico de las obras, caracterizado generalmente por el eclecticismo, así como la terminología, los valores, ejemplos y oposición de esta forma verbal, en especial, con el pretérito perfecto simple canté. Destacamos a partir de la investigación la necesidad de prestar atención al enfoque teórico y metodológico en la práctica de ELE para la obtención de un mayor rendimiento didáctico. 
Palabras clave: Imperfecto. Gramática. ELE. Cantaba. Metodología.

\begin{abstract}
The aim of this article is to analyze the treatment of imperfect tense in a corpus constituted by 24 Spanish grammars of a foreign language (ELE) of different levels and languages published between 1988 and 2015. We investigate, in particular, the theoretical framework of the works, generally characterized by the eclecticism, as well as the terminology, values, examples and the opposition of this verbal form, especially with the simple past canté. We emphasize from the research the need to pay attention to the theoretical and methodological approach in the practice of ELE in order to obtain a greater didactic performance.
\end{abstract}

Key Words: Imperfect tense. Grammar. ELE. Cantaba. Methodology.

\title{
1. INTRODUCCIÓN
}

El estudio teórico de materiales didácticos en la enseñanzaaprendizaje de lenguas debe ser un instrumento evaluativo de sumo interés y preocupación para los profesionales de este ámbito del conocimiento. Enseñar una lengua no es simplemente cuestión de uso (uso de la lengua) o de materiales dinámicos y aparentemente pedagógicos. Enseñar una lengua supone transmitir al discente un instrumento de comunicación, de concepción y de entendimiento del mundo. Por ello, un buen método de enseñanza-aprendizaje de una lengua debe partir, necesariamente, de un concepto de lengua y de un constructo teórico (aunque sea mínimo) de cómo debe diseccionarse y explicarse dicha lengua a partir de un contexto y una situación pragmática dada.

Sobre esta hipótesis construimos la base del presente artículo, conscientes de la necesidad de abordar el sustento teórico de los materiales didácticos, en este caso, de español como lengua extranjera (en adelante, ELE). Solo así se podrá enseñar de forma adecuada y coherente el español o cualquier otra lengua.

Este trabajo, además, se inscribe en un proyecto más amplio sobre el sistema verbal español y su tratamiento en gramáticas y materiales/manuales de ELE que pretende ofrecer al profesorado y alumnado un conjunto de reflexiones y directrices sobre cómo se aborda, en cada uno de estos 
textos, una de las categorías más ricas y complejas del sistema lingüístico español: el verbo. En, aproximadamente, dos años pretendemos presentar un volumen completo de todas las unidades de la conjugación española con el análisis detallado, en cada manual/gramática, de nomenclatura o terminología, ejemplos, modelo teórico de base, perspectiva de análisis, tipo de enfoque, contraste entre unidades verbales, usos prototípicos y no prototípicos (según la forma verbal estudiada), etc.

Así pues, este trabajo pretende contribuir, desde el ámbito de la teoría lingüística y su contraste en un corpus concreto, a la amplia producción y perspectivas de análisis de los diversos aspectos relacionados con el español como lengua extranjera, lo que supone una potenciación de la que fue línea pionera en los estudios sobre lingüística aplicada: la enseñanzaaprendizaje de lenguas, de acuciante actualidad y demanda en una sociedad cada vez más multicultural y polilingüe.

\section{OBJETIVOS}

En un interesante y útil trabajo, Susana Pastor Cesteros (2000) parte de cinco aspectos fundamentales de la relación entre teorías lingüísticas actuales y enseñanza-aprendizaje de una lengua. En síntesis, son estos:

1. Cómo y en qué ha influido la teoría lingüística en la enseñanza de segundas lenguas en general y del español en particular.

2. Cómo puede aprovecharse en la actualidad, para la enseñanza del español, el rico bagaje de investigación teórica sobre las lenguas y su enseñanza-aprendizaje.

3. Presencia de la reflexión metalingüística en el contexto del aula.

4. La reflexión metalingüística como estrategia de aprendizaje del alumnado.

5. Necesidad de la teoría lingüística en la formación del profesorado de ELE.

Teniendo presentes estas ideas y en la convicción de que las relaciones entre teoría lingüística y enseñanza de segundas lenguas resulta no solo necesaria sino muy útil y fructífera, hemos construido los objetivos de este artículo:

1. Contribuir al análisis teórico de los materiales de ELE.

2. Ayudar en la selección de manuales y materiales.

3. Apoyar la idea de la importancia del enfoque teórico y metodológico 
en la práctica de ELE.

4. Enfatizar las necesarias y útiles relaciones entre teoría/práctica de ELE y teorías lingüísticas.

Contribuimos, en definitiva, a corroborar la idea de Pastor (2000: 39) en la que afirma:

Una opinión generalmente aceptada y de la que a menudo oímos hablar coincide en considerar que existe una distancia enorme (e injusta, podemos añadir), entre lingüística y enseñanza de segundas lenguas: parece que los contenidos teóricos que se enseñan en la universidad están muy alejados de la posterior práctica docente [...] En realidad, sería conveniente que "estudiosos" y "enseñantes" de la lengua tuvieran los suficientes puntos de contacto como para que se beneficiaran de sus respectivos conocimientos unos de otros (entre otras cosas porque, aunque con distintos matices, la mayoría de los "estudiosos" enseñan y la mayoría de los "enseñantes" estudian). Partimos de la base de que en todo buen profesor de idiomas una sólida formación lingüistica es inseparable del bagaje didáctico; la utilidad que tenga después una determinada teoría lingüistica o gramatical dependerá de la aplicación que se haga de ella o, lo que es lo mismo, de la habilidad didáctica $y$ de la sensibilidad del profesor para extraer de ella cuanto considere que puede mejorar el aprendizaje.

\section{EL CORPUS}

\subsection{Referencia descriptiva}

Constituye nuestro objeto de estudio el análisis de un amplio corpus formado por veinticuatro gramáticas de ELE cuyos datos completos incluimos en el apartado de referencias bibliográficas, en concreto, en el de fuentes primarias. Hemos procurado que integraran este corpus aquellas gramáticas y aquellos autores o autoras que se suelen reconocer como referente en la metodología de la enseñanza-aprendizaje de ELE, como son Aquilino Sánchez Pérez (1991), Ángel López García (2005), Francisco 
Matte Bon (1995) o la Gramática básica del estudiante de español (2005), de la editorial Difusión, traducida a varias lenguas y que ha gozado desde su publicación de una excelente acogida tanto por parte del profesorado como del alumnado.

Por lo que respecta a las fechas de publicación, hemos procurado analizar gramáticas publicadas recientemente, de hecho es 2015 el año de algunas de ellas, como la Grammaire de l'espagnol moderne, cuyo autor es Jean-Marc Bedel (2015). La menos reciente de las analizadas es A new reference grammar of modern Spanish, cuyos autores son J. Butt y C. Benjamin (1988). Por último, una de las obras está publicada por el Instituto Cervantes, la Gramática práctica del español, cuya autora es María Victoria Pavón Lucero (2007).

Con el objetivo de que el corpus fuera lo más abarcador posible, entre las gramáticas que analizamos, si bien la mayoría están destinadas a estudiantes extranjeros sin especificar su lengua materna, han constituido también nuestro objeto de estudio gramáticas destinadas a los hablantes de algunas de las lenguas que más demandan el español, como son los hablantes italianos, ingleses y franceses. Así pues, seis gramáticas de nuestro corpus están destinadas de modo específico a su enseñanza, dos para cada grupo de hablantes. No obstante, determinadas obras como Actual. Gramática para comunicar en español, cuyos autores son Juan Carlos Barbero y Félix San Vicente (2006), o las dos gramáticas publicadas en Milán por María Lozano Zahonero $(2010 ; 2011)$, a pesar de que no lo anuncian en el título, prestan especial atención al alumnado italófono.

\subsection{Objetivos de las obras}

\subsubsection{Marco teórico y metodológico}

En la mayoría de las gramáticas analizadas, hay un predominio del eclecticismo como marco teórico y metodológico. Este rasgo ha caracterizado en la tradición tanto a las gramáticas como a los manuales de ELE. En efecto, si pensamos en el propio enfoque comunicativo, no podemos afirmar que esté basado en una teoría lingüística concreta, sino más bien en diversas teorías y con el objetivo, entre otros, de asumir que el lenguaje es un instrumento de comunicación y que, por lo tanto, no es suficiente conocer la gramática de una lengua para saber comunicarse con 
ella.

No obstante, aun partiendo de este presupuesto, las obras que estudiamos tienen como objeto principal el análisis de la gramática de la lengua española, sin que descuiden, en general, el principio de la lengua como instrumento de comunicación. Encontramos variación entre aquellas gramáticas de carácter más formalista, como las dos obras de María Lozano Zahonero: Gramática de referencia de la lengua española. Niveles A1-B2 y Gramática de perfeccionamiento de la lengua española. Niveles A1-B2 (Lozano Zahonero, 2010; 2011) y aquellas gramáticas con predominio de un análisis semántico, como las dos obras de Luis Aragonés y Ramón Palencia: Gramática de uso del español, niveles A1-A2 y niveles B1B2 (Aragonés/Palencia, 2009a; 2009b), o la Gramática explicada. Para niveles intermedios de Tarricone (Tarricone/Giol/González, 2012).

Por otro lado, varias de las obras de nuestro corpus se insertan en el marco teórico de la lingüística cognitiva y, por tanto, también dan importancia al componente semántico en el estudio de la lengua. En este grupo se incluyen la Gramática básica del estudiante de español, la obra coordinada por Alejandro Castañeda et al. (2014), titulada Enseñanza de gramática avanzada de ELE: criterios y recursos, o la Gramática cognitiva para profesores de español L2: cómo conciben los hispanohablantes la gramática de Ángel López García (2015). En cuanto a la importancia que conceden estas gramáticas al componente semántico, recogemos a continuación un fragmento tomado de la presentación de la Gramática básica del estudiante de español (Castañeda et al. (2014: 10): "Las características esenciales de la GBE son: explica el sistema gramatical español teniendo en cuenta siempre -tanto en la descripción gramatical como en los ejercicios- el significado y el uso real de los distintos recursos"1.

Por último, si nos fijamos en la estructura de estas obras, observamos que la mayoría de ellas organiza los contenidos partiendo de las categorías gramaticales o de los diversos tipos de oraciones, a partir de lo cual analizan su forma y su significado, con mayor o menor predominio de uno u otro componente, como ya hemos destacado arriba.

1. La negrita (que trasladamos a cursiva) aparece en el texto. 


\subsubsection{El destinatario}

Podemos distinguir de nuestro corpus aquellas gramáticas destinadas al alumnado de ELE en general o aquellas destinadas al profesorado, con independencia de que ambas puedan ser utilizadas por unos u otros. Entre las destinadas a la formación del profesorado, destaca la obra de M. ${ }^{a}$ Luz Gutiérrez Araus (2004), titulada Problemas fundamentales de la gramática del español como L2, así como la obra que ya hemos citado arriba Enseñanza de gramática avanzada de ELE: criterios y recursos. En el propio título indica Ángel López García el destinatario de su obra: Gramática cognitiva para profesores de español L2: cómo conciben los hispanohablantes la gramática (López García, 2015); así procede también Elisabet Areizaga Orube (2015) en Gramática para profesores de español como lengua extranjera. Por otro lado, en algunas de las obras los autores/ as o coordinadores/as indican explícitamente que están destinadas tanto al profesorado como al alumnado. Así lo afirma María Lozano Zahonero (2010: XIX) en sus dos gramáticas:

La obra está dirigida, en primer lugar, al estudiante universitario italiano, si bien esperamos que pueda resultar también de utilidad a un público más amplio, en particular a los estudiantes adultos que aprenden español de manera autónoma y a los profesores de español como lengua extranjera de cualquier nivel de enseñanza, quienes podrán encontrar aqui materiales e ideas para sus clases.

En otros casos, como la Gramática de referencia de español para italófonos, coordinada por Félix San Vicente (2013), se destina al profesorado de ELE para estudiantes italófonos y también a estudiantes italianos que tengan una alta competencia en español.

Analizando unas obras y otras, constatamos que las destinadas al profesorado suelen incorporar un mayor componente teórico explícito, mientras que las destinadas al alumnado, si bien lo presuponen, suelen plantearse como objetivo la descripción de la gramática que le resulta válida a todo aquel que aprende español, y no la reflexión teórica sobre la lengua.

Por otro lado, como ya hemos afirmado en el $\S 3.1 .$, nuestro corpus 
está constituido tanto por gramáticas cuyos destinatarios son el alumnado o el profesorado de ELE en general (11 y 4 gramáticas; 45,83\% y 16,6\%, respectivamente), por gramáticas destinadas tanto al profesorado como al alumnado de $\operatorname{ELE}(4 ; 16,6 \%)$, como por las dirigidas a estudiantes cuya lengua materna es el italiano, el francés o el inglés ( 5 gramáticas; $20,83 \%$ ). Al incluir estas últimas obras, nuestro objetivo ha sido abarcar un corpus extenso y cuyos objetivos fueran diversos; las destinadas al alumnado italófono, francófono y anglófono son, además, gramáticas de carácter contrastivo. Recogemos, a continuación, en un gráfico los datos que acabamos de exponer:

Gráfico 1

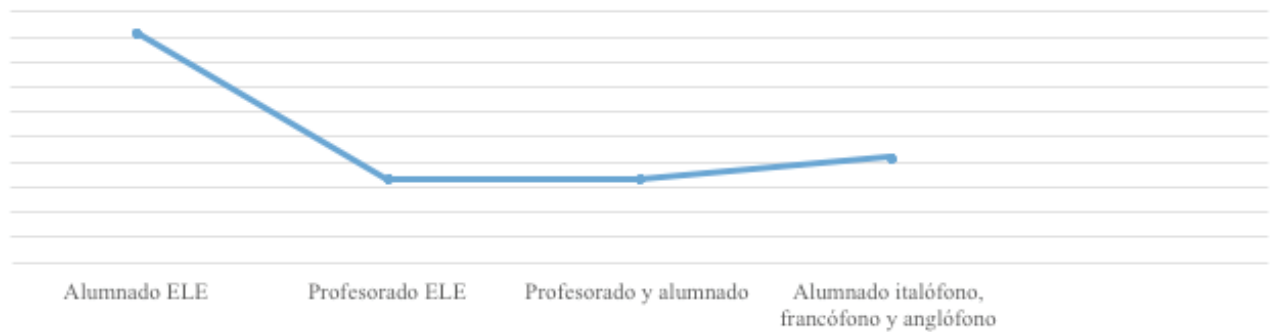

El destinatario

\subsubsection{Los niveles}

Forman nuestro corpus gramáticas de todos los niveles de enseñanzaaprendizaje fijados por el Marco común europeo de referencia para las lenguas. La mayoría de las obras hacen referencia a los niveles establecidos por el Marco, desde A1 hasta $\mathrm{C} 2$, si bien algunas hablan de niveles inicial, intermedio y superior. Por otro lado, hemos constatado que las gramáticas destinadas al alumnado especifican con más frecuencia los niveles de lengua que las destinadas al profesorado, que, en general, abarcan todos los niveles o bien están destinadas a los niveles superiores. 


\section{EL IMPERFECTO DE INDICATIVO: VALORES PROTO- TÍPICOS Y NO PROTOTÍPICOS}

A lo largo de la tradición bibliográfica, muchos han sido los trabajos dedicados a la forma verbal del español cantaba. En ellos destacamos, por un lado, la atención a los valores que en Zamorano/Martínez-Atienza (2014) denominábamos prototípicos y que se corresponden con los rasgos de tiempo pasado o presente del pasado y aspecto imperfecto o imperfectivo tradicionalmente asignados a esta unidad verbal. Por otro lado, la atención a los valores que etiquetábamos como no prototípicos, esto es, aquellos usos en los que el valor temporal y aspectual expresado no corresponde con el de tiempo pasado y aspecto imperfectivo; se trata de valores como el llamado imperfecto de cortesía, imperfecto narrativo, imperfecto lúdico, etc.

En la tradición que va desde Nebrija (1492) hasta Amenós, Leonetti y Morgado (2012) dos han sido las constantes con relación a cantaba (vid. Zamorano Aguilar, 2013):

a) El tipo de terminología utilizada para hacer referencia a los valores no prototípicos.

b) La explicación que se ofrece en los distintos trabajos al hecho de que el pretérito imperfecto, frente a otros tiempos como el pretérito perfecto simple o indefinido, manifieste significados que no corresponden al valor temporal de pasado ni al aspectual de imperfecto.

Por lo que respecta a la terminología, constatamos la tendencia a considerar secundarios los valores no prototípicos del pretérito imperfecto. Observamos que frente a los usos rectos, se habla de usos dislocados, frente a los usos reales, de usos irreales, frente a los valores primarios, de valores secundarios.

En cuanto a la explicación ofrecida, con frecuencia se presenta una descripción de los distintos significados modalizados del pretérito imperfecto, más que la justificación de que estos se manifiesten. A menudo, los usos modales se entienden a partir de los rasgos de los usos prototípicos. Así, el carácter aspectual imperfectivo, de acuerdo con el cual los límites inicial y final del evento no se focalizan, explicaría que el tiempo verbal objeto de nuestro estudio presentara determinados usos no referidos al pasado, sino al presente, al futuro o al futuro del pasado, o cuyo 
carácter no fuera factual ${ }^{2}$. Frente a ello, otros tiempos como el pretérito perfecto simple, de aspecto aoristo, no permiten tales usos. Observamos esta explicación incluso en autores que no defienden el valor aspectual imperfectivo para el pretérito imperfecto, como Gutiérrez Araus (1995).

\subsection{Valores prototípicos}

El análisis de la tradición anterior a 1950 (Zamorano Aguilar, 2013) arrojó las siguientes conclusiones de interés: 1) la nomenclatura asienta valores temporales (pasado) o aspectuales (imperfecto) en el Renacimiento, por influjo de la tradición latina (Prisciano); 2) el archilexema de cantaba es pretérito imperfecto y se gramatiza en el Anónimo de 1555; 3) se pueden distinguir tres etapas de evolución: i) gestación, en el siglo XVI; ii) seguimiento (1565-1830), conservación de las etiquetas renacentistas y reorganización de los valores conceptuales; iii) tradición e innovación (1830 en adelante), mantenimiento de las etiquetas anteriores y, guiados por la influencia de Port-Royal y la tradición racionalista francesa (Condillac, Destutt, Beauzée, Girard, entre otros), se introducen nombres que materializan la hipótesis temporal, a partir de la dicotomía tiempos absolutos/tiempos relativos.

Desde el punto de vista conceptual, el proceso de gramatización de cantaba se ha venido estableciendo sobre canté: a) el 48,21\% de los tratados que se analizaron en Zamorano Aguilar (2013) se acoge a la hipótesis temporal de diferenciación de ambas formas; b) en algunos casos se observa la defensa de una hipótesis vaga tempo-aspectual, como consecuencia de factores externos a la propia constitución de la obra: receptor (extranjeros, alumnado de primaria o secundaria, etc.), naturaleza del texto (escolar, comercial, pedagógico, etc.), entre otros; c) se observa una pervivencia absoluta de las tres hipótesis fundamentales: temporal, aspectual y tempo-aspectual.

En la tradición posterior a 1950, y por lo que se refiere al aspecto imperfectivo, encontramos discrepancia entre los distintos autores. Por un lado, figuran aquellos que consideran que el pretérito imperfecto expresa aspecto imperfectivo $\mathrm{y}$, por tanto, mantienen que el contraste entre esta

2. En este mismo sentido, se ha hablado también de la indeterminación del pretérito imperfecto, que permitiría la diversidad de usos modalizados, a diferencia de otros tiempos como el pretérito perfecto simple o indefinido. Véase al respecto el trabajo de Bazzanella (1990). 
forma y el pretérito perfecto simple es aspectual, ya que la segunda expresa un evento concluido y del que se focalizan sus límites (aspecto aoristo), mientras que el pretérito imperfecto expresa un evento del que se focaliza una parte (aspecto imperfectivo) ${ }^{3}$. Una postura distinta la mantienen los autores que defienden que entre ambas formas la diferencia es temporal: el pretérito perfecto simple expresa anterioridad del evento con respecto al momento de la enunciación y el pretérito imperfecto simultaneidad con respecto a un evento o momento temporal anterior al momento de la enunciación. Este análisis se desarrolla en la línea de Bello (1847-1860), quien habla, para el pretérito imperfecto, de copretérito por expresar simultaneidad con respecto a un pretérito. En esta segunda línea se sitúan los trabajos de Rojo (1974), Gutiérrez Araus (1995) o Rojo y Veiga (1999). El resto de los autores sostiene que el valor de copretérito es uno de los que puede expresar el pretérito imperfecto, si bien no el único.

Otro de los rasgos característicos del pretérito imperfecto es el carácter relativo o anafórico, esto es, el hecho de que no se oriente directamente con respecto al momento de la enunciación, sino a otra referencia anterior. De nuevo, contrasta con el pretérito perfecto simple, de carácter deíctico y, por tanto, orientado con anterioridad al momento de la enunciación. Junto a la oposición deíctico-anafórico, encontramos la de tiempo absoluto (junto a canté, para las formas canto y cantaré) y tiempo relativo primario o relativo propiamente dicho (junto a cantaba, cantaría, he cantado, habré cantado y hube cantado) (Marcos Marín, 1981).

Debido a las características tempo-aspectuales del pretérito imperfecto, se ha presentado con frecuencia como tiempo por excelencia para la descripción y para la expresión de eventos durativos. Así, cuando la narración se presenta en pretérito imperfecto "resulta más demorada, sugestiva y minuciosa, frente al corte que el indefinido impone" (Alcina y Blecua, 1955: 796).

Tanto la oposición entre descripción y narración como la de acción secundaria frente a acción principal no parecen corresponder con la que establecen aquí el pretérito imperfecto y el pretérito perfecto simple, que, en ambos casos, desde el punto de vista del aspecto léxico o modo de acción, expresan eventos de carácter puntual.

3. Alarcos (1994) utiliza la terminología terminativo-no terminativo para referirse a este contraste aspectual entre el pretérito perfecto simple y el pretérito imperfecto. 


\subsection{Valores no prototípicos}

La historia lingüística española anterior a 1950 explica que la gramatización de los valores modales de los tiempos del verbo se produce, según los datos del corpus estudiado en Zamorano Aguilar (2013), en las primeras décadas del siglo XIX, específicamente, con Calleja (1818). El Renacimiento y el siglo XVIII han sido los siglos de la gramatización formal y de la gramatización conceptual de los valores rectos (básicos) temporales y aspectuales.

Los valores modales que se han descrito y analizado a partir de 1818 son los siguientes: narrativo, futuridad ${ }^{4}$, supratextualidad ${ }^{5}$, oblicuidad $^{6}$, entre otras?

Por su parte, la crítica posterior a 1950 recoge y explica los siguientes valores no prototípicos: narrativo, de conato, de cortesía (valor de presente), condicional o de pospretérito, de opinión, hipotético, lúdico o imaginativo y contrafactual. En Zamorano/Martínez-Atienza (2014) defendimos que estos usos del imperfecto pueden ser explicados mediante la que hemos denominado hipótesis como focalizador de la enunciación. El imperfecto, en numerosos casos, se emplea para formalizar el evento de una enunciación presente y el valor que adquiere en estas secuencias es el de focalizador de toda una enunciación pasada (o varias), para lo cual neutraliza sus valores tempo-aspectuales.

De esta manera, podemos afirmar que los múltiples valores que la tradición y la crítica actual han asignado al imperfecto se pueden concentrar en dos. Por un lado, valores prototípicos de tiempo pasado y aspecto imperfectivo; por otro, valores no prototípicos como focalizador de la enunciación.

4. El valor de futuridad lo encontramos por vez primera en nuestra tradición en Noboa: este singular valor del imperfecto se localiza por vez primera en el corpus en Noboa (1839: 84). La tradición marca la futuridad de cantaba bien con relación al momento de la enunciación, bien con relación a un momento anterior al de la enunciación.

5. Bello (1847-1860) intuye un valor supratextual o, simplemente, textual que permite el empleo del copretérito en un juego de movimientos más amplios que los estrictamente oracionales.

6. El imperfecto hace las veces de otras formas verbales (oblicuidad) pero, con frecuencia, no se explican ampliamente las razones de ese intercambio de unidades.

7. Otros autores, sobre todo del XX, se refieren a muchos más valores del imperfecto, en un intento de sistematización semántico-nocional, sobre ejemplos concretos, del contenido gramatical que puede condensar el imperfecto. 


\section{ANÁLISIS DE LOS DATOS}

\subsection{Terminología}

La terminología empleada para rotular la unidad cantaba suele estar bastante consensuada en el corpus analizado. Los datos cuantitativos (véase Gráfico 2) reflejan la presencia de términos tradicionales junto a términos de acuerdo general. Así, la etiqueta copretérito solo la emplea De Molina Redondo (y, de forma indirecta, Butt y Benjamin, 1988) ${ }^{8}$ y, además, conjuntamente con pretérito imperfecto, nombre del que dice que es tradicionalmente (De Molina Redondo, 2011: 107) considerado así. El empleo de la nomenclatura de Andrés Bello es una muestra de prestigio (histórico) ante una modificación de las tradicionales etiquetas en donde primaban los valores temporales de las formas y el contraste entre ellas. La metodología de ELE se hace, pues, eco de esta innovación en el terreno de la terminología técnica en lingüística.

Gráfico 2

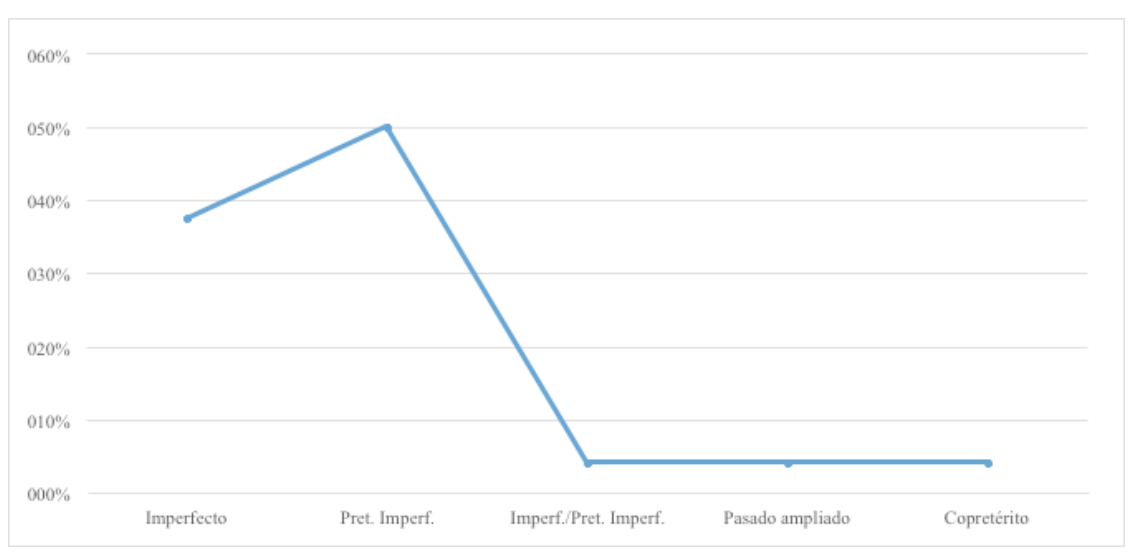

Terminología de cantaba

8. Estos autores utilizan el rótulo imperfect pero también hacen referencia a cómo se denomina la unidad cantaba en otros textos: pretérito imperfecto (la RAE y Seco) y copretérito (Bello). También indica que otras gramáticas inglesas lo denominan imperfect: Ramsey/Spaulding (1958) y Harmer/Norton (1957), como vía para reforzar su propuesta terminológica en textos para anglófonos. 
Solo en el caso de López García (2015) el empleo de la etiqueta pasado ampliado supone una manera de recoger aquellos valores que el autor considera, desde el ámbito de la lingüística perceptiva, prototípicos o, al menos, más exclusivos y excluyentes de la forma. En este caso, López García (2015) apuesta por el valor que en Zamorano/Martínez-Atienza (2014) denominamos focalizador de la enunciación y cuyo rasgo más característico es su proyección hacia el presente.

El consenso con relación a las etiquetas imperfecto y pretérito imperfecto se manifiesta, por ejemplo, en el uso indistinto de ambos términos que se hace en Barbero y San Vicente (2005), aunque el valor prototípico destacado en esta obra para cantaba es el aspectual.

El $87,5 \%$ de los textos del corpus emplean o imperfecto $(37,5 \%)$ o pretérito imperfecto (50\%). En el caso de imperfecto se ha observado que suele ser más empleado por textos que defienden el valor aspectual o tempo-aspectual de cantaba como valor prototípico. Por su parte, pretérito imperfecto se emplea en aquellos textos que tienen una consideración semántico-temporal o tempo-aspectual (nunca exclusivamente aspectual) de cantaba con relación a sus valores prototípicos.

Así pues, podemos establecer las siguientes tendencias con respecto a la terminología que se observa en el corpus analizado:

1. Las etiquetas que nuestros gramáticos emplean suelen ser transparentes con relación al valor que otorgan, como más destacado o prototípico, a la forma cantaba.

2. El uso de imperfecto suele reflejar valores aspectuales o tempoaspectuales, nunca exclusivamente temporales.

3. El uso de pretérito imperfecto suele plasmar valores temporales o tempo-aspectuales, nunca exclusivamente aspectuales.

\subsection{El contraste (o no) de cantaba con otras unidades del sistema verbal español}

El análisis del corpus revela, en primer lugar, que la unidad cantaba es claramente una unidad relativa y que se explica y justifica en relación con otras unidades del sistema verbal español; por ello, el 91,6\% de los tratados/gramáticas estudiadas incluyen en la descripción de cantaba algún tipo de contraste con otras formas del verbo. Solo en dos textos (Bedel, 2015 y Gerboin/Leroy, 2015), que suponen el 8,4\% del corpus, 
no establecen stricto sensu oposición con ninguna otra forma del sistema; en cambio, sí abordan las posibles sustituciones que se pueden realizar, funcional y semánticamente hablando, entre cantaba y otras unidades ${ }^{9}$, sobre todo, para referirse a valores no prototípicos. Resulta sintomático que ambos textos sean gramáticas contrastivas español-francés, las dos únicas de nuestro corpus.

En la mayoría de los casos, pues, se establece contraste con otras unidades del sistema, como acabamos de indicar. Pueden distinguirse, en ese contraste, tres situaciones distintas:

a) Se establece con una sola unidad del sistema.

b) Se contrasta con varias formas del verbo.

c) Se establece una jerarquía en el contraste que implica lo que podemos llamar (1) contraste prototípico (en una primera fase explicativodescriptiva); (2) contraste no prototípico o secundario (en una segunda fase). De esta manera, cantaba queda bien analizada en primera fase y, además, bien delimitada en segunda.

Gráfico 3

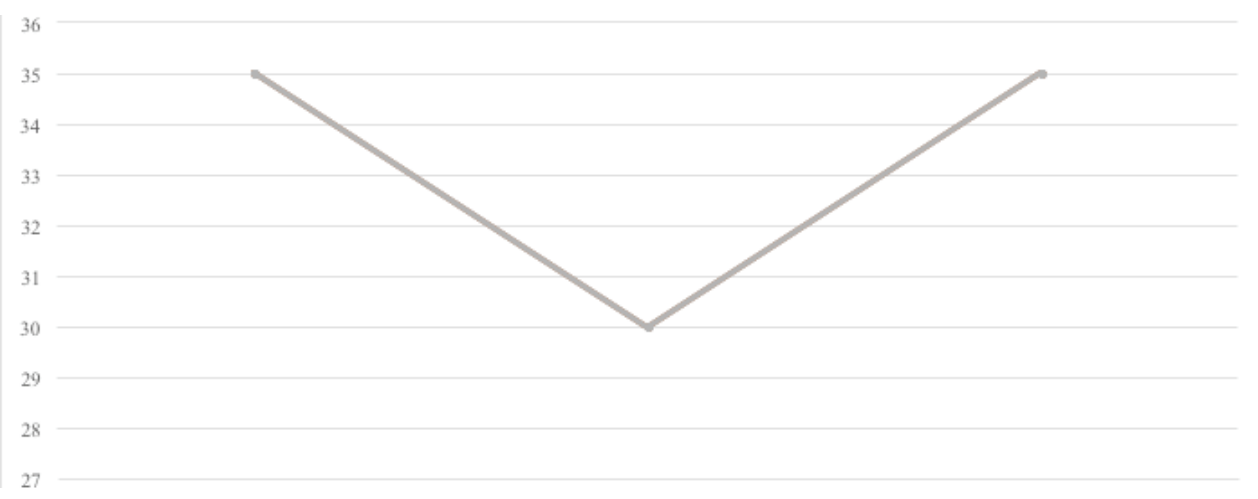

Tipo de contraste de cantaba y otras formas del sistema verbal español

Veamos, por grupos, las conclusiones del análisis:

9. Nos referimos a intercambios funcionales (en ambas gramáticas los mismos) entre el imperfecto y el condicional o el presente de indicativo. En los dos casos, nos encontramos ante textos que abordan los valores de cantaba de manera individualizada, aunque sí abordan otro tipo de contrastes, como, por ejemplo, canté-he cantado. 
a) Cuando el contraste se realiza con una sola forma del sistema dicha unidad es, en el 100\%, de los casos con canté. Los valores del contraste, aunque se analizan más detalladamente en el apartado 4 de este artículo, podemos señalar que se centran en contenidos (1) semántico-temporales, (2) tempo-aspectuales o (3) de tipo aspectual (cantaba-canté: acciones pasadas no acabadas $v s$. acciones pasadas acabadas).

b) Si el contraste se realiza con varias formas, los valores que se manifiestan en el contraste son, preferentemente, de tipo aspectual:

b1) Aspecto durativo vs. aspecto no durativo: cantaba vs. cantél he cantado (San Vicente, 2013: 121; Sánchez/Sarmiento, 2008: 114-115; Lozano Zahonero, 2010: 285; Lozano Zahonero, 2011 51-52; Sánchez Pérez, 1991: 537-541).

b2) Pasado de la descripción vs. pasados de la acción: cantaba vs. perfectos (Areizaga, 2015: 26-28).

b3) Tiempos absolutos vs. tiempos relativos, es decir, sitúan la acción con relación al momento de la enunciación vs. con relación a un momento distinto al de la enunciación. La unidad cantaba sería un tiempo relativo (Pavón, 2007: 114).

c) Por último, un $35 \%$ de los tratados abordan el contraste desde una consciente o inconsciente jerarquía. Es lo que hemos denominado jerarquía de contraste. Así, la mayoría de estos textos (el 85,7\%) establece un primer nivel de contraste (primera fase o contraste prototípico) con la unidad canté; el resto $(14,3 \%)$ lo lleva a cabo con todo el sistema verbal español.

Por lo que respecta al segundo nivel de contraste (segunda fase o contraste no prototípico), se realiza con: indef.);

unidades perifrásticas con imperfecto (estaba + gerundio $v s$. pret.

canté (en el 14,3\% del total);

había cantado, y en un tercer nivel con canto;

habia cantado, solamente;

he cantado;

canto;

cantaré.

Los valores de dicho contraste suelen ser de tipo aspectual o tempoaspectual, como se explica en el apartado 4 de este estudio. 
Por último, si analizamos con qué unidades del sistema (de forma global) se contrasta la unidad cantaba, los resultados que muestra la investigación del corpus son:

Gráfico 4

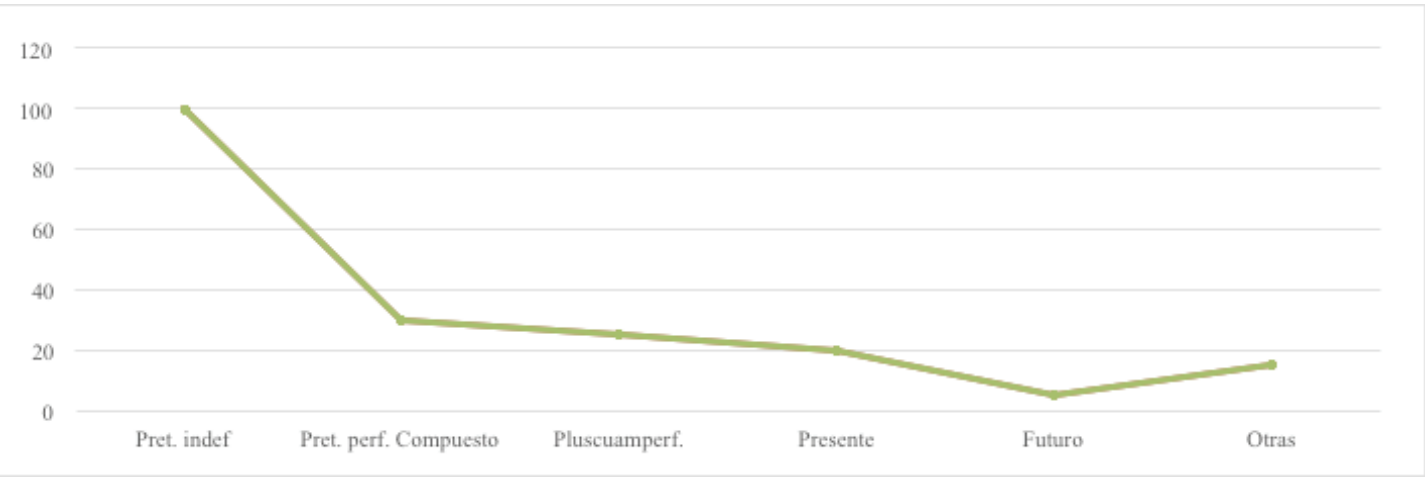

Unidades del contraste

El contraste en estos casos se basa en la propia concepción prototípica o no prototípica de los valores asignados, como abordamos seguidamente.

\subsection{Valores prototípicos}

En la tradición investigadora sobre el pretérito imperfecto, se ha estudiado casi siempre este tiempo verbal en contraste con otro tiempo también simple de pasado, a saber, el pretérito perfecto simple o pretérito indefinido. Las teorías sobre las diferencias entre ambos tiempos se pueden sintetizar en dos:

1) La teoría temporal, de acuerdo con la cual las diferencias entre el pretérito imperfecto y el pretérito perfecto simple se basan, exclusivamente, en el tiempo gramatical. Los estudiosos de esta línea caracterizan el pretérito imperfecto como un presente del pasado, o, siguiendo a Andrés Bello (18471-18605), como copretérito. En esta teoría se sitúan, entre otros, destacados estudiosos como Guillermo Rojo (1974) o Alexandre Veiga (2008).

2) La teoría tempo-aspectual, de acuerdo con la cual las diferencias entre ambos tiempos se basan en la categoría del aspecto. Según esta teoría, el pretérito perfecto simple se caracteriza por expresar un evento de 
aspecto perfectivo, esto es, el evento completo, mientras que el pretérito imperfecto se caracteriza por expresar aspecto imperfectivo, y por tanto por mostrar una parte del evento, pero no los límites inicial y final. En esta segunda línea se sitúan también estudiosos del verbo en español como Ángeles Carrasco Gutiérrez (2000) o Luis García Fernández (1998).

En el corpus de gramáticas que constituye nuestro objeto de estudio, la mayoría sigue la hipótesis tempo-aspectual y, por lo tanto, caracteriza el pretérito imperfecto como forma de pasado imperfectiva o no terminativa. No obstante, vemos reflejada también la hipótesis temporal en obras como Gutiérrez Araus (2004: 49), quien sostiene que

La forma cantaba, caballo de batalla del aprendizaje del Español como Segunda Lengua, creemos que queda perfectamente explicada sobre la base de la categoría temporalidad, sin necesidad de implicar al aspecto verbal y considerarla imperfectiva, como se ha venido haciendo $y$ aún se hace en gran parte de las gramáticas de la lengua española.

En esta segunda teoría se sitúan también las dos gramáticas de Luis Aragonés y Ramón Palencia (2009). El resto de las obras analizadas explican el pretérito imperfecto y su relación con el pretérito perfecto simple según criterios tempo-aspectuales.

Entre los valores semánticos característicos que con mayor frecuencia destacan las distintas gramáticas y que consideramos prototípicos, figuran la descripción de eventos en pasado o la expresión de eventos habituales.

Por otro lado, gramáticos como López García (2005) definen el pretérito imperfecto como pasado ampliado. Otros como Matte Bon (1995) caracterizan este tiempo por su estatismo, frente al dinamismo del pretérito perfecto simple.

Junto a la relación con el pretérito perfecto simple, es frecuente que en la caracterización del pretérito imperfecto se haga referencia a su relación con el presente, que, como ya hemos señalado arriba, mostraba Bello. Así, destaca Castañeda (2014: 287):

¿De dónde procede entonces la habitual interpretación no terminativa? La respuesta tiene que ver con la concepción 
implícita en el sistema esbozado hasta ahora. El IMPERFECTO, en esencia, es el equivalente al PRESENTE pero en un espacio no actual (al igual que el CONDICIONAL cantaría lo es respecto del FUTURO cantará). Esta idea se resume con la fórmula usada antes de 'presente de entonces'. [...] Asi las cosas, el carácter no teminativo prototípico del IMPERFECTO es equivalente al carácter no terminativo prototípico del PRESENTE, pero en la esfera del pasado o lo no actual.

Así pues, nuestra investigación demuestra que en el corpus existe un claro predominio de la explicación tempo-aspectual de la forma cantaba y de su contraste con canté:

Gráfico 5

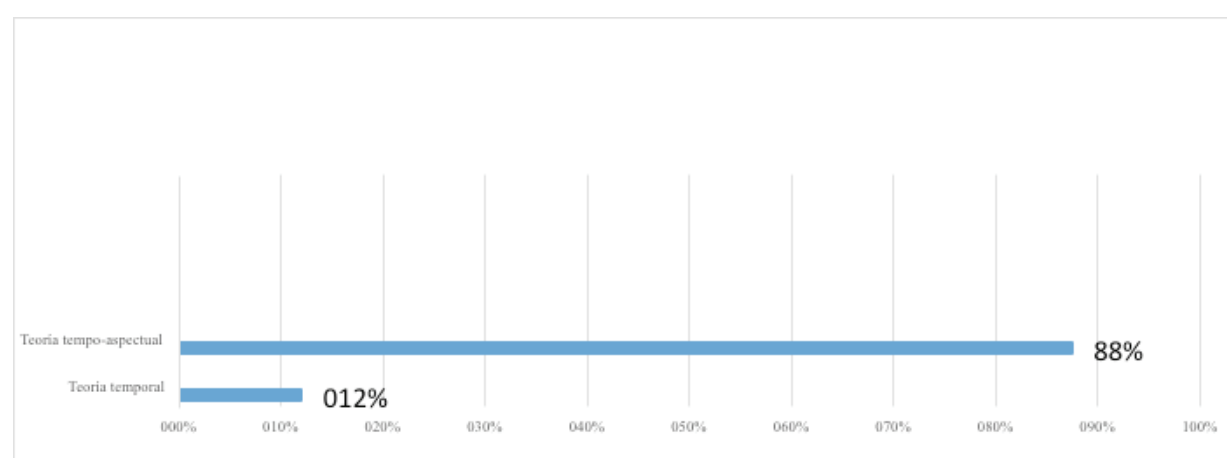

Teorías para explicar cantaba en el corpus de la investigación

\subsection{Valores no prototípicos}

En casi todas las gramáticas que hemos analizado, encontramos referencia a los valores que denominamos no prototípicos del pretérito imperfecto. Cabe destacar que buena parte de los autores, al hacer referencia a ellos, establecen relaciones con otros tiempos verbales, como veíamos con los valores prototípicos. En particular, con el condicional, con el futuro y con el presente. El ejemplo más claro de ello en nuestro corpus lo constituye la obra de Gutiérrez Araus (2004), que clasifica estos valores no prototípicos según criterios temporales. Así pues, distingue entre 1) imperfecto en contexto de presente; 2) imperfecto en contexto 
de futuro hipotético e 3) imperfecto en contexto de pasado de narración principal. A su vez, en 1) incluye 1.1) discurso anterior presupuesto; 1.2) imperfecto de cortesía e 1.3) imperfecto de fantasía. Entre los valores de 2), destaca: 2.1) expresar un deseo y 2.2) expresar condicionalidad y concesión. Comprobamos, pues, que los distintos valores modalizados se describen partiendo de sus significados temporales.

Por otro lado, en varias de las obras analizadas encontramos la referencia a la relación que el pretérito imperfecto mantiene con el condicional. Así lo reflejan Barbero/San Vicente (2006: 340):

En la expresión común, el imperfecto, dada su condición de tiempo del pasado, puede sustituir al condicional simple, sin el matiz hipotético común de esta forma verbal:

- Para expresar una acción futura en relación con el pasado: Ayer Margarita me dijo que venía hoy a vernos. Pues yo le entendí que no venía (en lugar de vendría en ambos casos).

- Para dar un consejo: Yo que tú, iba a la fiesta, no lo dudaría. Yo en tu lugar, no me lo pensaba.

- También sustituye al condicional simple en la apódosis (oración principal) de las oraciones condicionales poco posibles con si: Si pudiera, me marchaba ahora mismo para Barcelona. ¡Cómo me gustaría! Si me llamaran, claro que iba con ellos a Cuenca.

La relación con el condicional también figura en Butt/Benjamin (1988), en Barbero/Bermejo/San Vicente (2010), en Bedel (2015), en Gerboin/Leroy (2015), así como en De Molina Redondo (2010: 109), quien destaca la alternancia del pretérito imperfecto con la forma en -ría para la expresión de deseos: “¿Yo? Me \{tomaría/tomaba\} una cerveza grande y me \{quedaría/quedaba\} en la gloria”. La volvemos a encontrar en Sánchez Pérez (1991: 120), donde se señala el registro coloquial de este uso del pretérito imperfecto con valor condicional: "Si tuviera dinero, compraba esta casa (=compraría)". Lieberman (2008: 119) se refiere también a este valor y habla del significado de Pro-futuro en casos como "Quedé con una amiga en que íbamos al cine". Además, destaca la aparición de este tiempo en apódosis de período condicional: "Si tuviera dinero, te lo compraba". 
Es también frecuente la referencia a la relación con el futuro, como comprobamos en Bedel (2015), en Lieberman (2008), en Sánchez/ Sarmiento (2008) o en Lozano Zahonero (2011: 51), quien destaca el uso prospectivo irreal si indica "que el evento es contrario a la realidad", así como el valor de atenuación si "presenta una información atenuada, que no se recuerda con claridad: Esta tarde había una conferencia, pero la han suspendido; Pedro llegaba mañana, ¿no?’. Este mismo valor lo destaca también Pavón (2007: 115): “La fiesta era mañana, ¿no?”

La relación del pretérito imperfecto con el presente la encontramos con frecuencia en varias de estas gramáticas al describir los valores no prototípicos. Así aparece en Aragonés y Palencia (2009), en De Molina Redondo (2011) o en San Vicente (2013), quien afirma que el pretérito imperfecto puede expresar acciones presentes cuando queremos pedir algo con cortesía. De modo similar figura en Lieberman (2008: 119) al hablar del valor de cortesía y del lúdico de este tiempo:

Los dos valores que siguen denotan sucesos que ocurren en el momento del habla, pero que se enfocan alejados de su real actualidad: 3) De cortesía: se refiere al presente y se puede cambiar por el presente, pero con diferente grado de cortesía. Se us a con unos pocos verbos, por ejemplo: llamar, molestar, venir, buscar, traer, querer, desear, necesitar. (c) Te llamaba para pedirte los apuntes. 4) Lúdico. Lo usan los niños para describir los escenarios de sus juegos. El imperfecto acentúa su carácter de ficción: d) Entonces vos estabas prisionero y yo te rescataba.

También Matte Bon (1995: 28) se refiere a esta relación con el presente al afirmar que:

[e]n relación con el presente cronológico, se emplea para neutralizar parcialmente el carácter remático de la información y presentarla como algo que ya estaba en el aire: - ¿Tú dónde vas a pasar el verano? - Pues pensaba irme a Canadá a ver a mi hermana. El empleo de este tiempo es una estrategia que el enunciador emplea muy a menudo para no mostrarse demasiado decidido, no afirmar 
con demasiada energía su yo, y parecer, en cierta medida, más dispuesto al diálogo, más disponible.

Por último, entre los valores modales del pretérito imperfecto a los que se refieren los gramáticos (en la mayoría de los casos sin hablar explícitamete de modalidad), los más frecuentes son el valor de cortesía, el lúdico y el narrativo o periodístico.

\subsection{Los ejemplos}

En este último apartado de nuestra investigación pretendemos analizar la conceptualización prototípica que, en los textos de ELE estudiados, se realiza de la forma cantaba a través de las primeras ejemplificaciones de esta forma verbal, ya que partimos de la hipótesis de que el ejemplo, como recurso didáctico y explicativo fundamental en la enseñanza-aprendizaje de lenguas, pretende reflejar el valor representativo (en nuestra terminología, prototípico) de la unidad en cuestión, en nuestro caso, el pretérito imperfecto.

En primer lugar, el estudio del corpus ha revelado dos conceptualizaciones tipificadas:

a) Valor individualizado o aparentemente individualizado.

b) Valor en contraste con otras formas verbales.

Así, el 30\% de los tratados estudiados conceptualizan de forma prototípica la unidad cantaba a partir del contraste con otras unidades del sistema verbal español; por su parte, el $70 \%$ acomete una conceptualización inicial de forma individualizada y explicita valores diversos de tipo temporal, aspectual o tempo-aspectual.

Por lo que respecta al contraste (véase también 4.2 en este trabajo), conviene destacar la presencia de tres situaciones teóricas diferentes:

1. Textos en los que se refleja de forma clara la conceptualización a través del contraste ( $20 \%$ de los tratados). Esta realidad la encontramos en Barbero/San Vicente (2006), Aragonés/Palencia (2009a; 2009b), De Molina Redondo (2011) y Lieberman (2008). En todos los casos el contraste inicial se realiza con canté y el valor prioritario es tempo-aspectual, es decir, simulteneidad con un evento anterior al momento del habla: 
(1) El otro día estaba yo/él/ella/Manuel/Sofía haciendo la compra en el supermercado $y$, de repente, hubo un intento de atraco (Barbero/San Vicente, 2006: 338).

(2) Cuando llegué, hacía mucho frio/Lo he leido mientras esperaba mi turno (De Molina Redondo, 2011: 107).

En todos los casos, además, se realiza también una caracterización indirecta de tipo semántico-temporal (narración vs. descripción en el pasado).

2. Obras en las que se establece una jerarquía de nivel (5\%) entre el contraste y la conceptualización individualizada, lo que deja patente la -quizá- necesidad pedagógica de concretar esta jerarquización en el aula de ELE. Esta situación teórico-metodológica nos la encontramos en tratados que gradúan el nivel de lengua, por ejemplo, Lozano Zahonero (2010; 2011). En Lozano Zahonero (2010) se realiza una conceptualización inicial de cantaba de forma individualizada y tempo-aspectual (como acción que transcurre en el pasado y en la que no se precisa ni principio ni fin de dicha acción). Por su parte, en Lozano Zahonero (2011) se aborda la conceptualización inicial desde el contraste: "Pedro subía las escaleras con esfuerzo/Pedro subió las escaleras con esfuerzo", donde se pone de relieve la concepción aspectual de la oposición cantaba vs. canté.

3. Tratados en los que se aborda ambiguamente el contraste (5\%). Esta situación es difícil de delimitar pero podría observarse en Barbero/ San Vicente (2006: 338): el texto, al menos indirectamente, es consciente y refleja el carácter descriptiva y pedagógicamente dual de cantaba:

(3) El otro día estaba yo/él/ella/Manuel/Sofía haciendo la compra en el supermercado y, de repente, hubo un intento de atraco.

En la explicación no se aborda el contraste de forma directa, pero el ejemplo lo recogen, en este caso, para mostrar la morfología de cantaba. De forma indirecta, se muestra el contraste, en tanto que la primera conceptualización que se realiza del imperfecto es, posteriormente, a través de Andrés, mi cuñado, era alto, que ejemplifica el valor semánticotemporal: descripción en el pasado ( $v s$. narración en el pasado con canté). 
Como hemos indicado al inicio de este apartado, el 70\% de los textos analizados acometen, en cambio, una conceptualización individualizada de cantaba a través de los primeros ejemplos. El estudio de dichos ejemplos refleja los siguientes valores prioritarios o prototípicos:

1. Acciones habituales en el pasado: Aragonés/Palencia (2009a; 2009b) y Bedel (2015).

2. Descripción en el pasado: San Vicente (2013) ${ }^{10}$ y Areizaga (2015).

3. Evento pasado:

3.1. no concluido: Matte Bon (1995) ${ }^{11}$ y Castañeda et al. (2014);

3.2. en su desarrollo, sin precisión de inicio o fin: Butt/Benjamin (1988), Sánchez Pérez (1991), Holton/Hadlich (2001), Sánchez/Sarmiento (2008), Lozano Zahonero (2010) y Tarricone/Giol/Seara (2012) y Gerboin/ Leroy (2015).

3.3. que se proyecta al presente: López García (2005). (2007).

4. Focalizador de la enuncación pasada: VV.AA. (2005) y Pavón

\section{Gráfico 6}

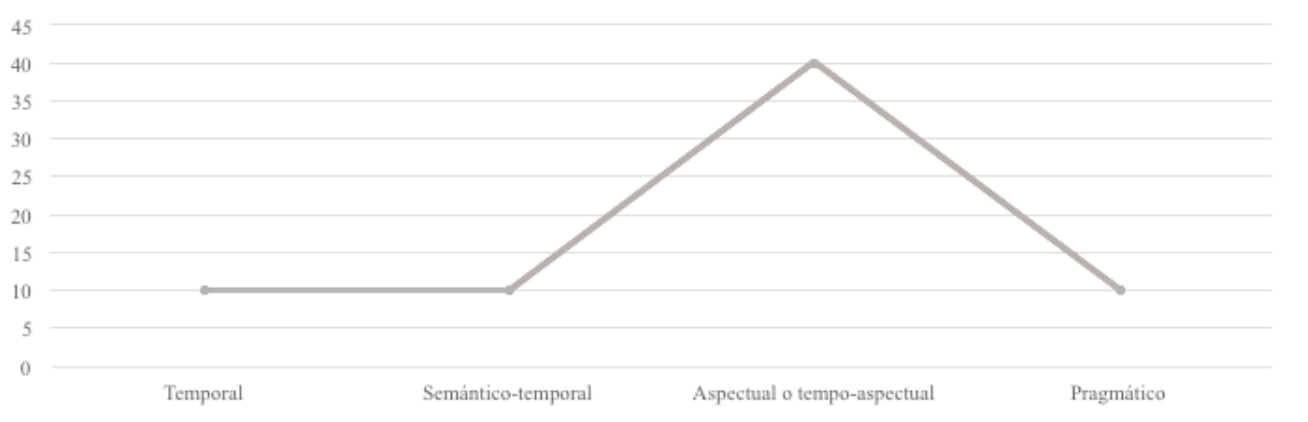

Valor de la conceptualización individualizada

Se observa, pues, que con los matices establecidos en 5.3 y en 5.4, la forma cantaba no recibe una conceptualización individualizada unánime,

10. Este texto, en realidad, comienza con el valor relativo de cantaba que "denota una situación que no se mide directamente desde el momento del habla" (San Vicente, 2013: 537); sin embargo, el primer ejemplo que se aporta es con estativos permanentes para inicios de texto. Podría, pues, considerarse una variante del valor descripción en el pasado.

11. En Matte Bon (1995) se habla, en realidad, no de acciones no concluidas como tal, sino de acciones que se repiten. Interpretamos que se trata de una variante del valor evento pasado no concluido. 
sino que se caracteriza de forma mayoritaria a través del valor aspectual o tempo-aspectual y, en cambio, de forma paralela en otros textos se destaca el valor temporal, semántico-temporal y pragmático ${ }^{12}$.

\section{SÍNTESIS Y CONCLUSIÓN}

El estudio y contraste realizado en este artículo sobre la unidad cantaba y su tratamiento en el corpus (amplio, variado, de autores y obras de referencia, de todos los niveles y de tres lenguas en contraste: italiano, francés e inglés) de gramáticas/manuales de español como lengua extranjera nos permite establecer las siguientes conclusiones:

1. La importancia de atender a las relaciones entre teoría y práctica en el aula de ELE, pues los muy poliédricos tratamientos que cantaba tiene en el corpus investigado vienen a apoyar la idea de la necesidad de prestar atención al enfoque teórico y metodológico en la práctica de ELE con el fin de extraer el máximo rendimiento no solo de la aplicación didáctica sino de los materiales con los que se trabaja.

2. Desde el punto de vista del marco teórico de los textos analizados, puede defenderse el predominio del eclecticismo con más o menos atención al componente semántico-formal ( $85 \%)$ o a los modelos cognitivos $(15 \%)$.

3. El estudio de la terminología empleada en el corpus arroja las siguientes tendencias:

- La etiqueta que nuestros gramáticos emplean suele ser transparente respecto al valor que otorgan, como más destacado o prototípico, a la forma cantaba.

- El uso de imperfecto suele reflejar valores aspectuales o tempoaspectuales, nunca exclusivamente temporales.

- El uso de pretérito imperfecto suele plasmar valores temporales o tempo-aspectuales, nunca exclusivamente aspectuales.

4. Por lo que respecta al contraste con otras formas del sistema verbal español, cantaba se manifiesta como unidad relativa: el 91,6\% de los gramáticos incluye contraste, privilegiando el que se establece con canté. Si dicho contraste se realiza con varias formas, los valores que suelen destacarse son aspectuales. El 35\% de los gramáticos establece

12. Este es el valor que nosotros hemos denominado focalizador de la enunciación (Zamorano/ Martínez-Atienza, 2014). 
lo que hemos denominado jerarquía del contraste, que pudiera tener repercusiones pedagógicas en el aula por lo que a graduación del proceso de enseñanza-aprendizaje se refiere.

5. Si abordamos de forma global los valores asignados a cantaba se perciben las siguientes posturas: a) valores prototípicos: en el $85 \%$ se sigue una hipótesis aspectual, esto es, pasado imperfectivo o no terminativo. Entre otros valores destaca la descripción de eventos y la expresión de eventos habituales; b) valores no prototípicos: se hace referencia a ellos con relación al valor temporal; por tanto, se contrasta el imperfecto con el presente, futuro y condicional. Los valores más frecuentes son: cortesía, lúdico y narrativo o periodístico.

6. Finalmente, hemos analizado los ejemplos que emplean los tratadistas del corpus para tipificar el valor canónico de cantaba. Se han consignado valores individualizados $(70 \%)$ y valores en contraste (30\%). En el caso del contraste tipificado: a) el 20\% recoge una conceptualización clara a través del contraste; b) el 5\% una jerarquía de nivel entre la conceptualización individualizada y la del contraste; c) el $5 \%$, finalmente, son tratados con ambigüedad en esta materia. En cuanto a la conceptualización individualizada, no se observa una tendencia unánime sino que se caracteriza de manera aspectual o tempo-aspectual $\mathrm{y}$, de manera paralela en ciertos tratados, de forma temporal, semánticotemporal y pragmática.

Los resultados obtenidos, por tanto, creemos que permiten abrir una necesaria vía de investigación que analice los textos didácticos actuales desde la perspectiva teórica y contrastiva, con el fin de seguir mostrando su rentabilidad para la didáctica de ELE y la confección adecuada, razonable y ajustada al funcionamiento real del español.

\section{REFERENCIAS BIBLIOGRÁFICAS}

ALARCOS LLORACH, E. (1994). Gramática de la lengua española. Madrid: Espasa-Calpe.

ALCINA FRANCH, J. y BLECUA, J. M. (1975). Gramática española. Barcelona: Ariel.

AMENÓS, J.; LEONETTI, M. y MORGADO, L. (2012). "Tiempos 
verbales y metarrepresentaciones: el caso del imperfecto". Comunicación presentada en el X Congreso de Lingüística General, Zaragoza, 18-20 de abril.

ARAGONÉS, L. y R. PALENCIA, R. (2009a). Gramática de uso del español. Teoría y práctica con solucionario. A1-A2. Madrid: SMHoepli.

(2009b). Gramática de uso del español. Teoría y práctica con solucionario. B1-B2. Madrid: SM-Hoepli.

AREIZAGA ORUBE, E. (2015). Gramática para profesores de español como lengua extranjera. Madrid: Ediciones Díaz de Santos.

BARBERO, J. C. y SAN VICENTE, F. (2006). Actual. Gramática para comunicar en español. Bologna: CLUEB.

BARBERO, J. C.; BERMEJO CALLEJA, F. y SAN VICENTE, F. (2010). Contrastiva. Grammatica della lingua spagnola. Bolonia: CLUEB.

BAZZANELLA, C. (1990). "Modal' Uses of the Italian indicativo imperfetto in a Pragmatic Perspective”. Journal of Pragmatics 14.3, 439-457.

BEDEL, J.-M. (2015). Grammaire de l'espagnol moderne. Paris: Presses Universitaires de France.

BELLO, A. y CUERVO, R. J. (1988). Gramática de la lengua castellana destinada al uso de los americanos. Ed. de Ramón Trujillo. Madrid: Arco/Libros, 1847-1860.

BUTT, J. y BENJAMIN, C. (1988). A new reference grammar of modern Spanish. London: Edward Arnold.

CALlEJA, J. M. (1818). Elementos de gramática española. Bilbao: Pedro Antonio de Apraiz.

CARRASCO GUTIÉRREZ, Á. (2000). La concordancia de tiempos. Madrid: Arco/Libros.

CASTAÑEDA CASTRO, A. et al. (coords.) (2014). Enseñanza de gramática avanzada de ELE: criterios y recursos. Madrid: Sociedad General Española de Librería.

DE MOLINA REDONDO, J. A. (2011). Gramática avanzada para la enseñanza del español. Edición de Pedro Barros García y Encarnación Morales Manrique. Granada: Universidad de Granada.

GARCÍA FERNÁNDEZ, L. (1998). El aspecto gramatical en la conjugación. Madrid: Arco/Libros.

GERBOIN, P. y LEROY, C. (2015). Grammaire d'usage de l'espagnol 
contemporain. Paris: Hachette, D.L.

GUTIÉRREZ ARAUS, M. L. (1995). Formas temporales del pasado en indicativo. Madrid: Arco / Libros.

(2004). Problemas fundamentales de la gramática del español como L2. Madrid: Arco / Libros.

HARMER, L. Ch. y NORTON, F. J. (1957). A Manual of Modern Spanish, 2. ${ }^{\text {a }}$ ed. London: University Tutorial Press.

HOLTON, J. S.; HADLICH, R. L. y GÓMEZ ESTRADA, N. (2001). Spanish grammar in review. Upper Saddle River: Prentice Hall.

LIEBERMAN, D. I. (2008). Temas de gramática del español como lengua extranjera: una aproximación pedagógica (2. ${ }^{\mathrm{a}}$ edición). Buenos Aires: Universidad de Buenos Aires.

LÓPEZ GARCÍA, Á. (2005). Gramática cognitiva para profesores de español L2: cómo conciben los hispanohablantes la gramática. Madrid: Arco / Libros.

LOZANO ZAHONERO, M. (2010). Gramática de referencia de la lengua española. Niveles A1-B2 según las directrices del Marco Común Europeo de Referencia para las Lenguas. Milán: Ulrico Hoepli.

(2011). Gramática de perfeccionamiento de la lengua española. Niveles C1-C2 según las directrices del Marco Común Europeo de Referencia para las Lenguas. Milán: Ulrico Hoepli.

MARCOS MARÍN, F. (1981). Curso de gramática española. Madrid: Editorial Cincel.

MATTE BON, F. (1995). Gramática comunicativa del español. Vol 1: De la lengua a la idea. Vol. 2: De la idea a la lengua. Madrid: Edelsa.

NEBRIJA, A. D. (1980 [1492]). Gramática de la lengua castellana. Ed. de A. Quilis. Madrid: Editora Nacional.

NOBOA, Br. A. M. (1839). Nueva gramática de la lengua castellana según los principios de la filosofía gramatical. Madrid: Impr. de don Eusebio Aguado.

PAVÓN LUCERO, M. V. (2007). Gramática práctica del español. Madrid: Espasa-Calpe (Colección Guías prácticas del Instituto Cervantes).

RAMSEY, M. y SPAULDING, J. K. (1958). A Textbook of Modern Spanish. New York: Henry Holt.

ROJO, G. (1974). "La temporalidad verbal en español". Verba 1, 68-149.

ROJO, G. y A. VEIGA (1999). "El tiempo verbal. Los tiempos simples". En Gramática Descriptiva de la Lengua Española, I. Bosque y V. 
Demonte (dirs.), cap. 44, 2867-2934. Madrid: Espasa-Calpe.

SAN VICENTE, F. (dir. y coord.) (2013). Gramática de referencia de español para italófonos. Bolonia: CLUEB, cap. XIX, 531-554.

SÁNCHEZ PÉREZ, A. (1991). Gramática práctica de español para extranjeros. Madrid: Sociedad General Española de Librería.

SÁNCHEZ PÉREZ, A. y SARMIENTO, R. (2008). Gramática práctica del español actual: español para extranjeros. Madrid: Sociedad General Española de Librería.

SARMIENTO, R. (1999). Gramática progresiva de español para extranjeros. Madrid: Sociedad General Española de Librería.

TARRICONE, L.; GIOL, N. y GONZÁLEZ SEARA, C. (2012). Gramática explicada. Para niveles intermedios con ejercicios + soluciones. Madrid: EnClave-ELE.

VEIGA, A. (2008). Co-pretérito e irreal / Imperfecto e inactual. El doble valor de la forma cantaba en el sistema verbal español y algunos problemas conexos. Lugo: Axac.

VV. AA. (2005). Gramática básica del estudiante de español. A1-B1. Barcelona: Difusión.

ZAMORANO AGUILAR, A. (2013). "Las formas simples del pasado del verbo en español: proceso histórico de gramatización". Romance Philolgy 67.1, 179-207.

ZAMORANO AGUILAR, A. y M. MARTÍNEZ-ATIENZA (2014). "Valores prototípicos y no prototípicos del pretérito imperfecto en español. Su hipótesis como focalizador de la enunciación”. En Simple and compound forms of the past in Spanish and other languages, S. Aspiazu (ed.), 179-194. Lugo: Axac.

Recibido el 9 de febrero de 2017.

Aceptado el 1 de marzo de 2017. 
